\title{
Numerical Simulation of Steel Target Penetration by Shaped Charge Distended Jet with a High-Polymer Liner
}

\author{
J. Y. Yi, ${ }^{1}$ Z. J. Wang, J. P. Yin, and B. H. Chang
}

School of Mechatronic Engineering, North University of China, Taiyuan, China

1yijianya513@126.com

This study investigates the characteristics of shaped charge jet formed with a high-polymer (PTFE) liner, as well as its penetration capabilities, by theoretical, experimental, and numerical methods. This work presents a viscoplastic model and equation of compressible fluid to describe the jet cohesive condition. It also shows that the high-polymer jet is inevitably distended. Two types of liner materials were studied: a high-polymer PTFE liner and a pure copper one. The corresponding numerical simulations of the two jet formations are presented. The pulse $X$-ray photographic technology was employed to observe the distended jet of the PTFE liner and the particulate jet of the copper one. The simulation and jet radiography results show that the two types of jet behavior with particulate and radial dispersion are ductile and related to the liner material. The distended jet formation result from the liner material was crushed at the high-pressure region because a sudden pressure jump induces the radial velocity rise, which results in lateral expansion. As compared with a typical copper penetration performance, the polymer distended jet had a larger aperture and lower penetration depth. Due to polymer liner lower density, this jet will have limited penetration as compared to that of a copper liner. The simulated results strongly agree with the experimental ones. The polymer material can be modified to obtain much better performance, which will greatly enhance the penetration capacity of polymer jets.

Keywords: explosive mechanics, shaped charge, high-polymer, jet.

Introduction. To produce longer and more stable jets, designers have used metal liner materials because metals have high density, high sound speed, good thermal conductivity, high dynamic fracture elongation properties, low viscosity, and low compression properties. These are necessary conditions for the formation of cohesive and stable jets. Most research about metal jets describe homogeneous dense metal materials that form the jet, and the penetration process through the constant ideal incompressible fluid theory and quasi ideal incompressible theory; this research is relatively mature. However, with the development of new materials, techniques, designs, and protective devices (composite, reactive, electromagnetic, and intelligent armors), there is a need for additional research. Moreover, the superior protection ability of the new protective devices challenges the traditional antiarmor weapons. Considering these factors, the improvement of damage performance of armor-piercing weapons has become a research focus in recent years.

Currently, research on liner material is divided into three categories: pure metal, multiphase composite material, and non-metal material. Pure metal liners are mainly produced from $\mathrm{Cu}, \mathrm{Ni}, \mathrm{Mo}, \mathrm{W}$, and $\mathrm{Ta}$ [1], while multiphase composite ones involve $\mathrm{W}-\mathrm{Cu}, \mathrm{Re}-\mathrm{Cu}$, and $\mathrm{Ta}-\mathrm{Cu}[2]$. Cowan and Bourne [3] studied oxide glasses as shaped charge liner materials due to their ability to stretch without the formation of necks that characterize jets produced from pure metal liners. Keeskes and Walters [4] studied the Zr-based bulk metallic glasses as shaped charge liner materials. The results showed that the liner behaved similar to particulate jets made from pressed powder liner materials. Jet formation was asymmetric and the particles in the jet were not uniform in size and dispersion. Baker et al. [5] used traditional silica-based glasses as shaped charge liner materials. The jet radiography results show the distinct regions with extremely particulate, ductile, or radically dispersed behavior. The resulting jet behavior appears to be both 
material- and design-dependent. The extreme particulate jet behavior appears to be related to the brittle nature of glasses observed at lower temperatures and pressures. Helte and Lundgren [6] empirically tested the penetration capability of precursors of tandem warheads against ERA (Explosive Reactive Armor), and found out that precursors with liners made of alumina powder, aluminum powder, and glass could penetrate but not detonate the ERA panels. In addition to the inorganic non-metallic materials, the current application of organic non-metallic materials (nylon, PTFE, and other polymer) has also been studied. Hirsch and Sadwin [7] showed that the firing of shaped charge liners made from a material of high compressibility, such as thermoplastic, produced cavities that expanded in diameter near the base of the cavity. This would have the potential to induce failure in brittle materials such as concrete. They showed that this effect was associated with short stand-offs of 1.5 cone diameters (CD) but no details were given of the liner material or cone angle. Haney and Wesson [8] described the use of polymer liners as an intermediate layer between a conventional metallic shaped charge liner and the explosive. The polymer would be forced into the cavity created by the metallic jet, where it would burn and decompose; the increased volume of combustion products would induce failure in the surrounding target material. Chang et al. $[9,10]$ studied the modified PTFE jet penetrating shell charge, which showed good efficiency at destroying shell charges. The perforation efficiency was enhanced by about $70 \%$ for the panel, and $30 \%$ for the back plate.

Clearly, because of the specific features of the above polymer, it has good penetration potential as the shaped charge liner material. The aim of the present work is to investigate the behavior of shaped jet charges that use high-polymer liners.

1. Governing Equations for Metal Jet Formation Processes. In the studies of metal jets, Birkhoff et al. proposed the classical steady jet theory. Later, Pugh et al. further developed the theory, proposing quasi-steady jet theory. The most important assumption of the initial jet formation theory is that the liner material is a non-viscous and incompressible fluid. This theory accurately describes the conical copper jet formation process. However, with the development and innovation of liner materials, these theories cannot accurately describe the jet formation of composite powder metal liners and non-metallic liners. The development of composite powder metal liner promotes the formation of shaped charge jet theory, and gives the effect of the liner material viscosity and compressibility to the jet formation.

So far, the viscoplastic model [11] basically does not include the non-steady state (non-constant) effect. The assumption is that the jet is regarded as an incompressible fluid, the other is regarded as a compressible fluid, and the material constitutive relation is based on the fully elastic-plastic strain hardening model. Godunov [12] studied the pressure model of the shaped charge liner, including the effect of the liner material viscosity on the jet formation. The jet formation standard basically does not consider the effects of the shock wave and the critical Mach number, but rather the metal liner viscoplastic properties. However, the standard shows that the jet is agglomerative rather than scattered or distended. That is to say that it has no radial velocity. Obviously, this model cannot accurately explain why the polymer jet is distended.

The compressibility effect can be ignored in the collapse process of the polymer liner. Applying the viscoplastic model, material viscosity can be expressed in the following form:

$$
R_{e}=\frac{\rho_{0} \varepsilon V \sin ^{2} \beta}{\mu(1-\sin \beta)},
$$

where $\rho_{0}$ and $\varepsilon$ represent density and thickness, respectively, $\mu$ is the dynamic viscosity coefficient, $\beta$ is the pressed angle, and $V$ is the flow velocity. When $\mu$ increases, $R_{e}$ will decrease. Meanwhile, the coefficient of the kinetic viscosity of polymer materials is much 
higher than that of metal materials, and the initial density is lower than that of metal materials. The Reynolds number relation of the two materials is given by

$$
R_{e p}<R_{e m},
$$

where $R_{e p}$ and $R_{e m}$ are the Reynolds numbers of the polymer material and metal, respectively. The fluid velocity associated with the Reynolds number is expressed as

$$
V^{*}=V\left(1-\frac{2}{R_{e}}\right)^{1 / 2} .
$$

where $V^{*}$ is the flow velocity related to the correlation coefficient of dynamic viscosity. According to the material viscosity coefficient, the velocity relationship is expressed as

$$
V_{p}^{*}<V_{m}^{*}
$$

In the cohesive collapse process of the liner initial stage, the polymer fluid velocity is lower than that of the metal because of the introduction of the dynamic viscosity coefficient. Given the conditions that the metal jet cohesive is $R_{e}>2$ and $R_{e p}<R_{e m}$, the polymer jet cannot form a coherent jet because it is more easily dispersed than the metal one when considering only the viscosity effect. As pressure increases, the liner element will produce the radial velocity, which leads to lateral expansion after they converge into the high-pressure region. Hence, the liner material compressibility cannot be ignored.

Sound speed relates to the compression performance of the medium. Sound speed will be lower when the compressibility of the medium is higher. The sound speed of the polymer material is much lower than that of the metal material, so the polymer material compressibility is better than that of the metal material. The Bernoulli equation of compressible fluid is expressed as

$$
\frac{P}{\rho}+e+\frac{u^{2}}{2}=\mathrm{const},
$$

where $e$ represents the fluid energy per unit mass. The above formula shows that in the one-dimensional case of entropic compressible fluid, the sum of pressure potential energy, kinetic energy, and internal energy in each section of fluid per unit mass is constant.

According to the continuity equation of compressible fluid,

$$
\rho u A=\text { const }
$$

where $\rho$ denotes fluid density, $u$ denotes fluid velocity, and $A$ denotes fluid width. The introduction of a streamline is named $l$, which is not close to the outer surface (Fig. 1). The relationship between the initial and flow states is given by

$$
\rho_{0} u_{0} A_{0}=\rho_{1} u_{1} A_{1}
$$

Here $\rho_{0}$ and $u_{0}$ denote the initial fluid density and velocity, respectively; $\rho_{1}$ and $u_{1}$ are the outflow fluid density and velocity, respectively; whereas $A_{0}$ and $A_{1}$ are the initial and outflow fluid width values, respectively. 


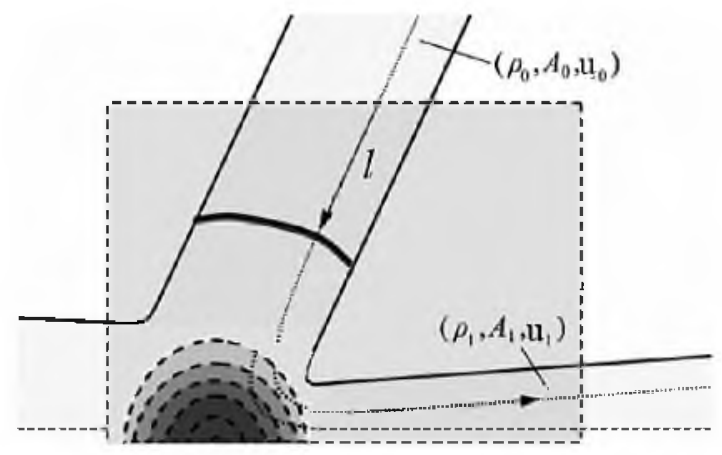

Fig. 1. Jet formation and the position of shock wave.

The compressible fluid energy equation is expressed as

$$
\frac{u_{0}^{2}}{2}+e_{0}+\frac{p_{0}}{\rho_{0}}=\frac{u_{1}^{2}}{2}+e_{1}+\frac{p_{1}}{\rho_{1}} .
$$

According to the viscous compressible fluid model, the streamline entropy is constant,

$$
T d s=d h-\frac{1}{\rho} d p=0
$$

where $s$ denotes entropy and $h$ denotes enthalpy. In addition, the momentum equation along the streamline is simplified as

$$
u d u+\frac{1}{\rho} d p=0 .
$$

Since the density is higher than zero, the enthalpy and pressure are the same, so the formula is given by

$$
\frac{d p}{d u}=-\rho u<0
$$

The above formula can be expressed as

$$
\frac{d \rho}{d u}=\left(\frac{\partial \rho}{\partial p}\right)_{s} \frac{d p}{d u}=-\frac{\rho u}{c^{2}}<0,
$$

where $c$ denotes the speed of sound. Along the streamline by mass flux density per unit area on the cross section of $\rho u$, then

$$
d(\rho u)=u d \rho+\rho d u
$$

The above equation can be expressed as

$$
\frac{d(\rho u)}{d u}=\rho\left(1-\frac{u^{2}}{c^{2}}\right)
$$


This shows that the mass flux density increases with fluid velocity in the subsonic speed, but in supersonic speeds, it exhibits the reverse trend.

In addition, the relationship between sound speed and fluid velocity can be expressed as

$$
\frac{d c}{d u}=\frac{1}{2 c} \frac{d c^{2}}{d u}=\frac{1}{2 c}\left(\frac{\partial c^{2}}{\partial \rho}\right)_{s} \frac{d \rho}{d u}=-\frac{\rho u}{2 c^{3}}\left(\frac{\partial^{2} p}{\partial \rho^{2}}\right)_{s}<0 .
$$

This shows that the sound speed decreases with fluid velocity. The relationship between the Mach number and fluid velocity is given by

$$
\frac{d M}{d u}=\frac{d}{d u}\left(\frac{u}{c}\right)=\frac{1}{c}+\frac{\rho M^{2}}{2 c^{3}}\left(\frac{\partial^{2} p}{\partial \rho^{2}}\right)_{s}>0 .
$$

This implies that the Mach number increases with fluid velocity.

The initial state of the flow end is equal to the unloading pressure in the high-pressure region, and $p_{0}=p_{1}=0$, because the dynamic viscosity coefficient of the polymer is very large, so the term $e$ cannot be ignored. The molecular theory of viscosity is very complex; it is seen as the viscosity of the molecular interaction movement of the friction resistant, so the end work flow overcomes viscosity resistance. The relationship between the initial state and the energy of the flow state is expressed as

$$
e_{0}>e_{1}
$$

In view of Eq. (8), we get

$$
u_{0}<u_{1}
$$

Equation (7) yields

$$
\rho_{0} A_{0}>\rho_{1} A_{1}
$$

Due to high compression performance of polymer materials, the relationship between the initial state and the density of the flow state is expressed as

$$
\rho_{0} \neq \rho_{1}
$$

The pressure and density decreases with fluid velocity, but if pressure is constant, the density decreases. The relationship between the initial state density and the flow state one is expressed as

$$
\rho_{0}>\rho_{1}
$$

From Eqs. (18) and (21), we get

$$
A_{0}<A_{1} .
$$

Therefore, the high-polymer jet is inevitably distended due to the effects of viscosity and compressibility.

2. Numerical Simulation of Jet Formation. The computational geometric model for the numerical simulation is shown in Fig. 2. Figure 2a is the schematic diagram of a shaped charge structure and Fig. $2 \mathrm{~b}$ is the finite element model. The initialization parameters are as follows: the liner cone angle is $60^{\circ}$, the diameter is $50 \mathrm{~mm}$, and the charge height is $65 \mathrm{~mm}$. 
The model consists of three components: the liner, explosive, and airflow. LS-DYNA-3D finite element software was used to numerically simulate the process when the jet was formed from high-polymer liner under the influence of blast loading. Meanwhile, the ALE algorithm, a representative of the unit algorithm, was used to simulate the detonation process of the explosives, the collapse of the liner, and the formation of the high-polymer jet. The ALE grid was sufficient to cover the detonation products and the formation space of the high-polymer jet. The $1 / 4$ model was established according to the symmetrical structure of the charge to save computing time.

The materials used in the numerical simulation are copper and PTFE, the material model is the elastic plastic fluid model, and the main material constitutive parameters are shown in Tables 1 and 2.

$\mathrm{T}$ a

Simulation Parameters of Warhead Explosive

\begin{tabular}{|c|c|c|c|c|c|c|c|c|}
\hline $\begin{array}{c}\rho, \\
\mathrm{g} / \mathrm{cm}^{3}\end{array}$ & $\begin{array}{c}D, \\
\mathrm{~m} / \mathrm{s}\end{array}$ & $\begin{array}{c}A, \\
\mathrm{GPa}\end{array}$ & $\begin{array}{c}B, \\
\mathrm{GPa}\end{array}$ & $R_{1}$ & $R_{2}$ & $\omega$ & $\begin{array}{c}E, \\
\mathrm{~kJ} / \mathrm{m}^{3}\end{array}$ & $\begin{array}{c}P_{C-J} \\
\mathrm{GPa}\end{array}$ \\
\hline 1.83 & 8480 & 748.6 & 13.38 & 4.5 & 1.2 & 0.38 & 9.0 & 36 \\
\hline
\end{tabular}

T a b 1 e 2

Simulation Parameters of Liner Materials

\begin{tabular}{|c|c|c|c|c|c|c|c|}
\hline Material & $\begin{array}{c}\rho, \\
\mathrm{g} / \mathrm{cm}^{3}\end{array}$ & $\begin{array}{c}G, \\
\mathrm{GPa}\end{array}$ & $\begin{array}{c}\sigma_{Y}, \\
\mathrm{GPa}\end{array}$ & $E_{h}$ & $\begin{array}{c}\text { Grüneisen } \\
\text { coefficient }\end{array}$ & $\begin{array}{c}C_{1}, \\
\mathrm{~m} / \mathrm{s}\end{array}$ & $S_{1}$ \\
\hline $\mathrm{PTFE}$ & 2.16 & 2.33 & 0.05 & 0.0364 & 0.90 & 1340 & 1.930 \\
$\mathrm{Cu}$ & 8.96 & 50.90 & 0.12 & 0 & 1.99 & 3940 & 1.497 \\
\hline
\end{tabular}

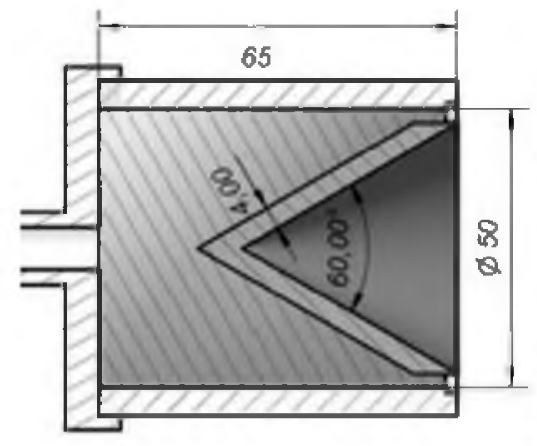

a

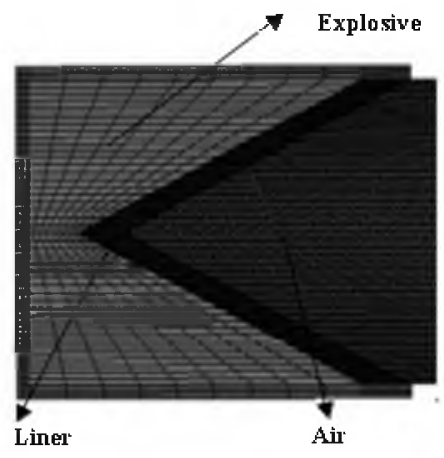

b

Fig. 2. Computational model: (a) schematic diagram of a shaped charge structure; (b) the finite element model.

To study the properties of polymer liner forming jets, two kinds of materials, PTFE (polymer) and copper (metal), were selected as the shaped charge liners, with a single point detonating charge at the center. Figure 3 shows the jet formation process of the PTFE and copper liner. As can be seen from Fig. 3a, the PTFE liner also can form an excellent ductile of the shaped charge jet. As compared with the typical copper jet patterns, the difference is more significant, with jet diameter as the most significant difference. The result of this distinction is that the radial velocity of the PTFE jet is too large, causing the jet radial distension in the tensile process. 


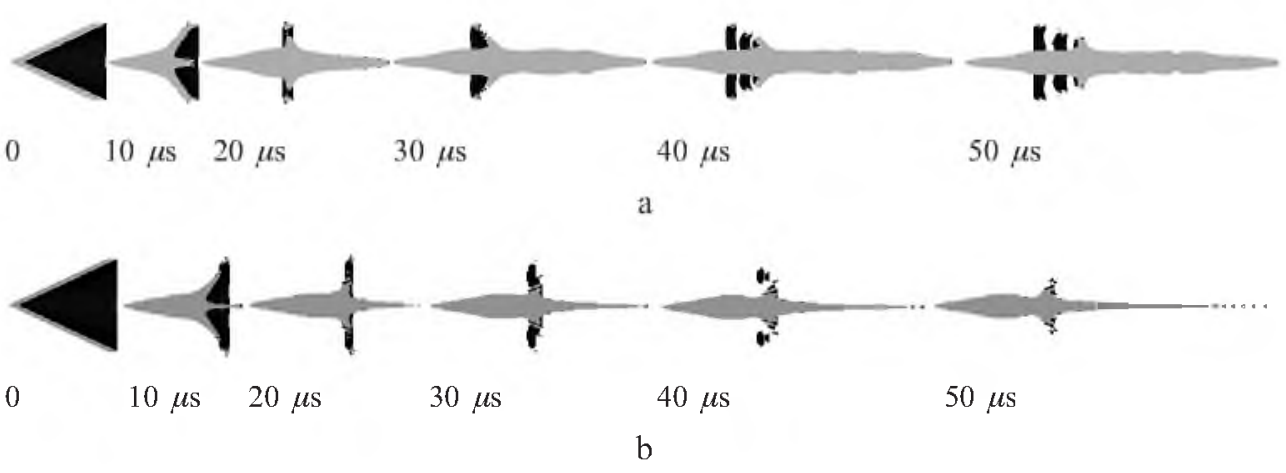

Fig. 3. The jet formation process of PTFE (a) and copper (b) liner shaped charges.

Analyzing the jet formation process of the two liner materials, the collapse of the PTFE liner cohere at $10 \mu$ s when the copper liner experiences the same variation. However, because of the different nature of liner materials, the PTFE belongs to the polymer liner materials and the copper belongs to the metal liner materials; the difference of the two jets in the patterns occurs at this moment. The PTFE jet had a distinctly larger diameter than the copper jet, which shows that the states of the two liners are the same in the collapse process, which can be collapsed with axial impact at certain speeds. The PTFE jet obtained a greater radial expansion velocity, which increases the jet diameter after the high-pressure disturbance region.

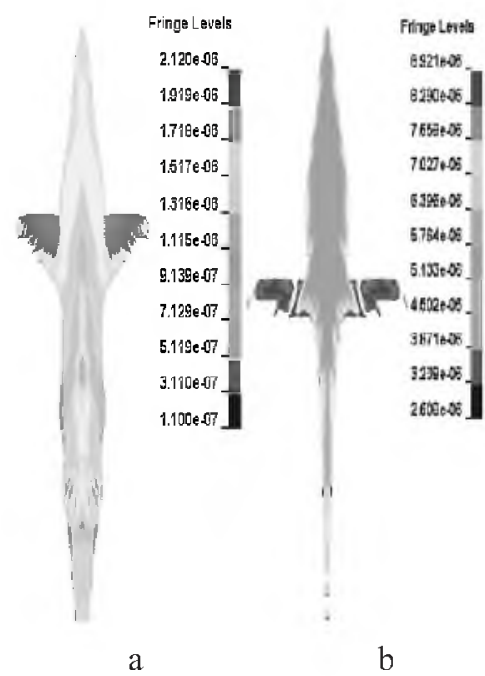

Fig. 4. The density distribution map of jets from charges with liners of (a) PTFE (a) and copper (b).

At $20 \mu$ s, the copper jet was continually stretched with the tip becoming more pointed. The PTFE jet had a continued radial expansion; it was also continually stretched because the polymer is ductile. At $30 \mu \mathrm{s}$, the head diameter of the PTFE jet became smaller, while the head of the copper jet broke into particles, and the rest of the cohesive jet still moved. As shown by the density distribution map (Fig. 4), the part of the intact (defect-free) copper jet was still a coherent jet, the jet density remained the same, the fracture jet particles had a lower density, and the PTFE jet had a much greater density near the axis. As the radial density distribution become smaller, the PTFE jet did not form the typical coherent jet 
under the influence of the radial velocity, but the expansion jet which was formed by uniform particle distribution. Over time, the copper jet head formed particles after continuous fractures, while the diameter of the PTFE jet remained unchanged, but the head of the PTFE jet became smaller. This distrubution map also shows that the slug of PTFE jet is similar to that of the copper one, which implies that the difference in the liner materials is related only to the jet shape, but not the slug shape if both charges have the same structure. Nevertheless, as shown by the jet density distribution chart, the copper jet slug maintained a full state while that of the PTFE jet dispersed into a particle state; nonetheless, the two parts were similar in shape. As a consequence of the non-negligible viscidity and compressibility of polymer, there were two different states.

Figure 5 shows radiographs of the observed jets, and the shaped charge structure used in the experiment is the same as the numerical simulations. The figure shows that the head diameter of the PTFE jet had a larger diameter, which showed the radial expansion state. However, the head diameter of the copper jet was very small, and the head of the copper jet formed a granular jet in the tensile process.

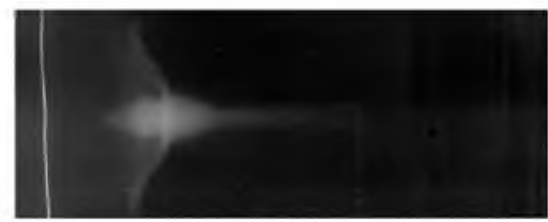

a

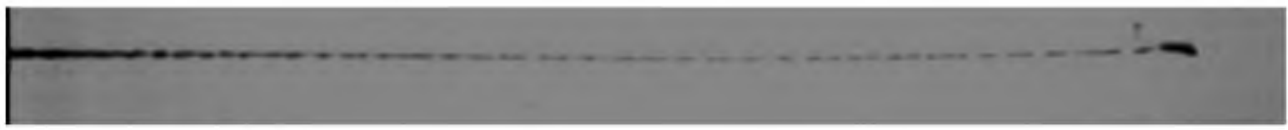

b

Fig. 5. Radiographs of jets from charges with liners of PTFE (a) and copper (b).

Figure 6 shows the state parameter graph of the jet when two different materials were used. Figure $6 \mathrm{a}$ is the graph of the head velocity curve of the jet, and Fig. $6 \mathrm{~b}$ is the graph of the radial velocity curve of the jet. Figure $6 \mathrm{c}$ is the graph of the length curve of the jet, and Fig. $6 \mathrm{~d}$ is the graph of the diameter curve of the jet.

Figure 6 a illustrates that the velocity of the copper jet is slowly decreased due to air resistance in the tension process. The velocity of the PTFE jet is identical to that of the copper jet in the formation stage jet collapse process. However, the velocity sharply declines in the tensile phase, due to high-speed particles of the distended jet consuming the formation energy by air resistance, and to continuous collision as a result of higher energy consumption.

Figure $6 \mathrm{~b}$ shows that the radial velocity of the PTFE jet reaches the maximum value when the jet flows in the process of the jet collapse, and is continually reduced during the stretching process. However, the radial velocity of the copper jet is very low, as compared to the PTFE one, and is gradually reduced, which affects the distended jet formation process.

Figure $6 \mathrm{c}$ shows that the jet length is the same at the initial stage, but the unstable fracture of the copper jet head in the tensile process results in a lower length growth rate for the copper jet as compared to that of the PTFE one. Nevertheless, the length of PTFE jet slowly decreases after a sharp increase due to jet velocity reduction.

Figure $6 \mathrm{~d}$ shows that the PTFE jet diameter is larger than that of copper one at the initial stage. With the increased the gap in stretching process, the diameters of the two jets reach their maximum values at the same time, and then are slowly reduced. 


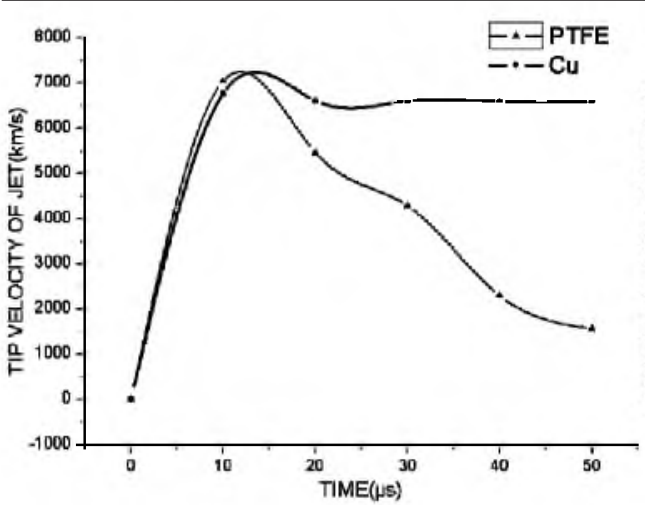

a

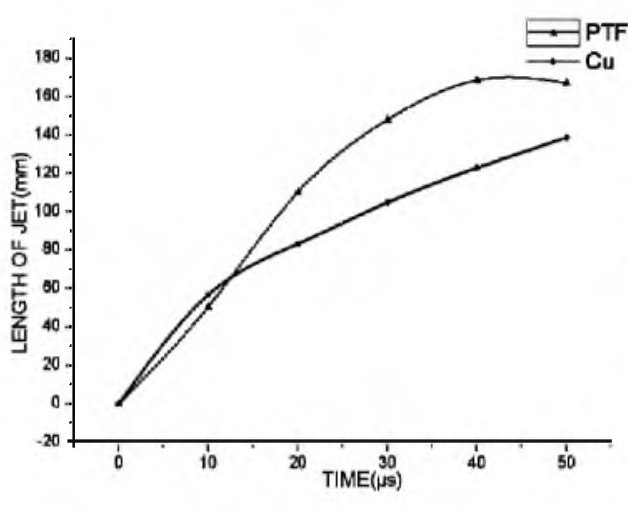

c

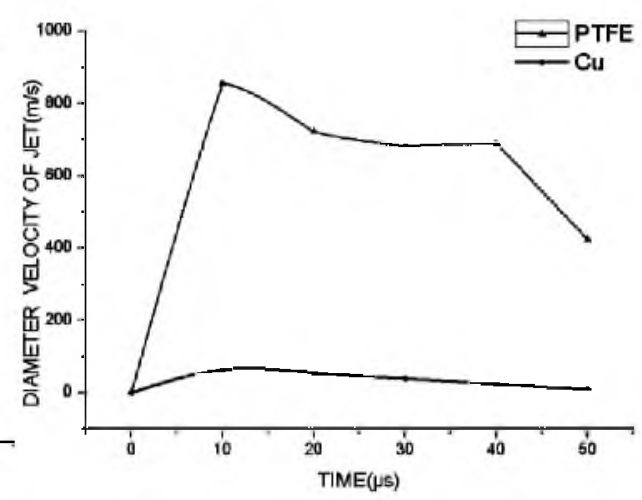

b

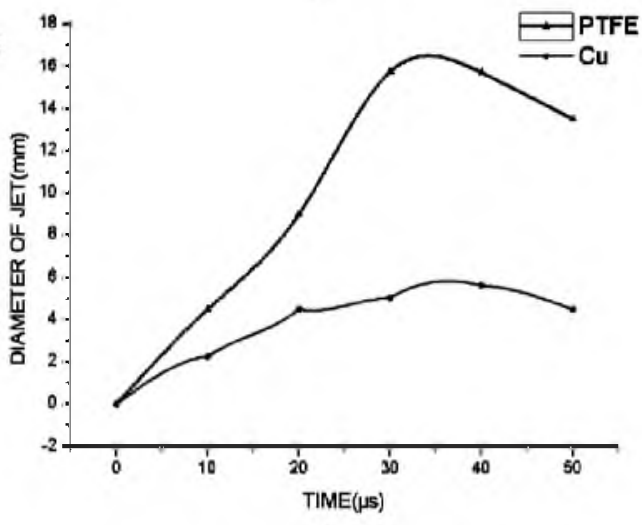

d

Fig. 6. State parameter graph of the jet: (a) the two jet tip velocity curves; (b) the two jet radial velocity curves; (c) the two jet length curves; (d) the two jet diameter curves.

In summary, the PTFE showed the greatest density of plastic materials in the polymer. As a liner material, PTFE can form distended jets, which is different from traditional coherent jets. The above research studied the shape particularity of distended jets by comparing their performance parameters to those of typical copper jets. The penetration performance of the polymer jet was studied by numerical simulation and experimental method, as follows.

3. Comparison of Numerical and Experimental Results. The shape of the polymer liner jet is different than that of a typical jet. In further study, the penetration performance of the polymer liner distended jet, the penetration experiment was designed and compared with the numerical simulation results.

Figure 7 shows the shaped charge used in the experiments. The distended jet properties must be known to determine the penetration depth and crater diameter of the shaped charge jets at $3 \mathrm{CD}$ standoffs.

The results obtained show that penetrations (crater depths) were higher for copper than for PTFE (Fig. 8). The penetration of the PTFE jet into the steel target was $14 \mathrm{~mm}$ and created a $20.9 \mathrm{~mm}$ diameter entry hole. The numerical simulation results gave a $11.8 \mathrm{~mm}$ penetration depth, and $18.61 \mathrm{~mm}$ crater diameter. In contrast, a copper liner with the same cone angle and standoff gave $30 \mathrm{~mm}$ penetration with a tapering hole and a $14.6 \mathrm{~mm}$ diameter entry hole. Numerical simulation gave a $24.87 \mathrm{~mm}$ penetration depth and an $8.4 \mathrm{~mm}$ crater diameter. 


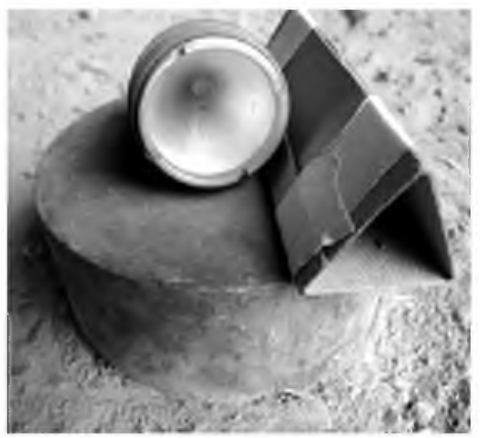

a

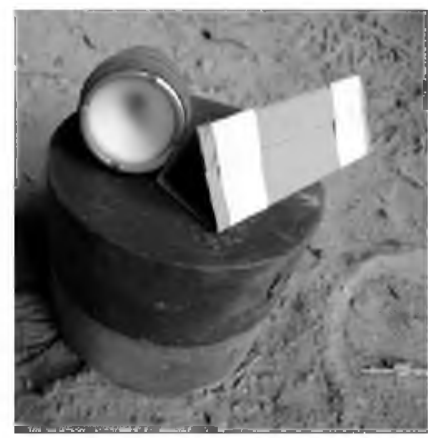

b

Fig. 7. The experimental arrangement of jet penetrating steel target: (a) copper; (b) PTFE.
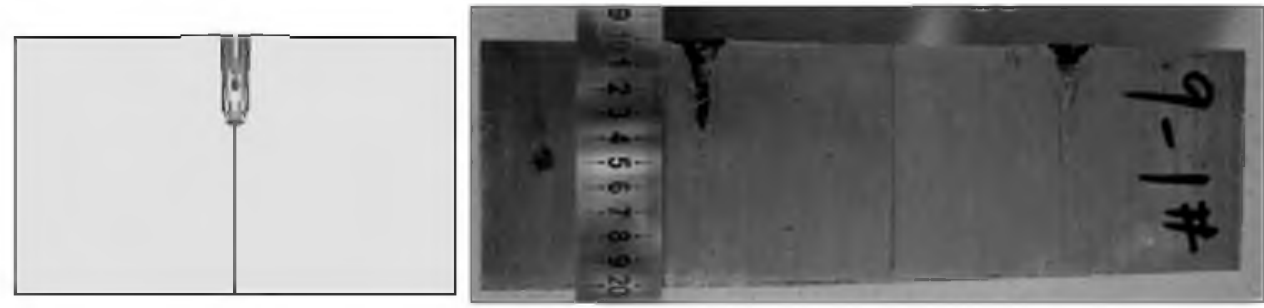

a
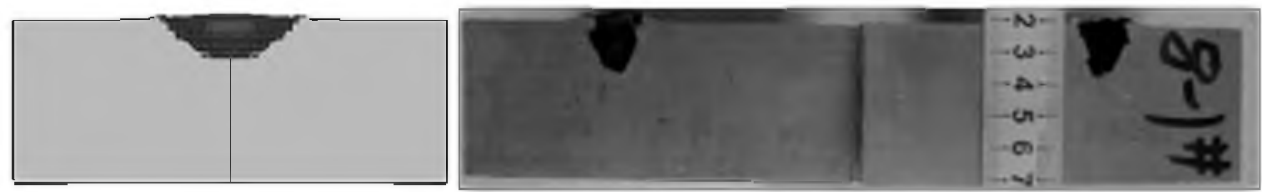

b

Fig. 8. The numerical and experimental results of the penetrating target: (a) copper; (b) PTFE.

The damage effect of the two jets in a steel target is different. The copper jet penetration depth is large, and the crater diameter is small. However, the PTFE jet penetration depth is small, and the crater diameter is large. However, its remarkable ability to produce large penetration holes is an advantage.

Conclusions. This study presents theoretical, numerical, and experimental examinations on the characteristics of jet formation with polymer liners, and their ability to penetrate steel targets. Two different liner materials (PTFE and copper) were studied.

1. Considering the liner material viscosity and compressibility, the application of the viscoplastic model fluid equations of the liner state and through the research on the liner into the crushed high pressure region, comparative analysis of outflow of metal jet and polymer jet. The cohesive condition of the polymer jet was obtained, which indicates that the charge structure is the same as that of the metal charge structure.

2. The simulated results of the jet formation with two different liner materials show that the PTFE liner produces a distended jet, while the copper one produces a particle jet. The numerical results agree with the radiography data.

3. This study presents the results of the numerical simulation and parameter analysis of the head velocity, radial velocity, length, and diameter evolutions of both jets. The initial stage of the PTFE jet exhibits a higher radial velocity, which causes a larger jet diameter. 
4. Numerical and experimental results show that the PTFE jet penetration into steel target displays a lower penetration depth and higher crater diameter than those of copper one. This suggests that the penetration capacity of the PTFE jet is influenced by the radial velocity, resulting in the enhanced radial expansion and deteriorated axial penetration abilities.

Acknowledgments. The authors would like to acknowledge the financial support from the Project supported by the National Natural Science Foundation of China under Grant No. 11572291.

1. C. Wang, J. X. Ding, and H. T. Zhao, "Numerical simulation on jet formation of shaped charge with different liner materials," Defence Sci. J., 65, No. 4, 279-286 (2015).

2. B. Zygmunt and Z. Wilk, "Formation of jets by shaped charges with metal powder liners," Propell. Explos. Pyrot., 33, No. 6, $482-487$ (2008).

3. K. G. Cowan and B. Bourne, "Oxide glasses as shaped charge liners," in: Proc. of the 21th Int. Symp. on Ballistics, Adelaide, South Australia (2004), pp. 419-423.

4. L. J. Keeskes and W. P. Walters, "Investigation of a bulk metallic glass as a shaped charge liner material," in: Proc. of the 23th Int. Symp. on Ballistics, Tarragona, Spain (2007), pp 31-38.

5. E. L. Baker, S. DeFisher, A. Daniels, et al., "Glass as a shaped charge liner material," in: Proc of the 26th Int. Symp. on Ballistics, Miami, FL (2007), pp. 340-347.

6. A. Helte and J. Lundgren, "Non-initiating precursor charge technology against era," in: Proc. of the 26th Int. Symp. on Ballistics, Miami, FL (2007), pp. 313-318.

7. E. Hirsch and L. Sadwin, "SDSC - a structure destroying shaped charge," in: Proc. of the 24th Int. Symp. on Ballistics, New Orleans, LA (2008), pp. 1110-1113.

8. J. Haney and D. Wesson, Shaped-Charge, US Patent US20050056459 A1 (2005).

9. B. H. Chang, J. P. Yin, Z. Q. Cui, and T. X. Liu, "Numerical simulation of modified low-density jet penetrating shell charge," Int. J. Simul. Model., 14, No. 3, 426-437 (2015).

10. B. H. Chang, J. P. Yin, Z. Q. Cui, and T. X. Liu, "Improved dynamic mechanical properties of modified PTFE jet penetrating charge with shell," Strength Mater., 48, No. 1, 82-89 (2016).

11. A. Chulya and K. P. Walker, "A new uniformly valid asymptotic integration algorithm for elasto-plastic-creep and unified viscoplastic theories including continuum damage," Int. J. Numer. Meth. Eng., 32, No. 2, 85-418 (1991).

12. S. K. Godunov, A. A. Deribas, and V. I. Mali, "Influence of material viscosity on the jet formation process during collisions of metal plates," Combust. Explo. Shock+, 11, No. 1, 1-13 (1975). 